\title{
Classical density functional approach to adsorption of hydrogen in carbon materials
}

\author{
V. V. Zubkov, V. M. Samsonov, I. V. Grinev, I. V. Popov \\ Tver State University, Tver, Russia \\ zvvictor@mail.ru
}

PACS 68.43.-h, 68.18.Fg, 68.43.De $\quad$ DOI 10.17586/2220-8054-2015-6-3-394-404

The adsorption of hydrogen in carbon adsorbents was investigated at low and high temperatures $(20.33,77$, 200 and $300 \mathrm{~K}$ ) over a wide range of pressures using the classical density functional theory. The adsorbent was simulated by a slit-like pore presented by the gap between two monocarbon (graphene) walls. In most cases, our results demonstrate a good agreement with the available experimental and theoretical results of other authors. A conclusion was made that, contrary to the low temperature region $(T<100 \mathrm{~K})$, at high temperatures (200 and $300 \mathrm{~K}$ ), predicted values for the adsorption and of the gravimetric density of hydrogen are not sufficient for the practical design of a hydrogen accumulator.

Keywords: Adsorption, hydrogen, carbon adsorbents, density functional theory.

Received: 6 April 2015

Revised: 21 April 2015

\section{Introduction}

For the last decade, the interest in the adsorption of hydrogen in carbon porous materials has peaked from time to time, first of all, in view of its possible applications in the hydrogen energetics. This kind of energetics may be considered as a very clear way for the production and storage of energy. Hydrogen is the most abundant element not only on the Earth but also in the whole Universe. It has the highest combustion temperature, and the only product of the hydrogen combustion is water. In the framework of the previouslymentioned problem, a specific, and most importantly, still unsolved issue concerns hydrogen storage. Some estimations [1] predict that $3.1 \mathrm{~kg}$ of the molecular hydrogen is required for a car to travel $500 \mathrm{~km}$ car mileage. At the same time, in [1] it is noted that the existing approaches to hydrogen storage do not allow one to solve the problem under consideration. For instance, using the compressed gaseous hydrogen is usually treated as one of the main alternatives to the liquefied hydrogen. However, the first of the above alternatives requires extremely high pressures, which is very dangerous. The storage of the liquefied hydrogen requires prohibitively high pressures as well as at very low temperatures. Among other alternatives, the use of pure and mixed metal hydrides should also be mentioned; however, these metal materials also do not allow one to solve the problem under consideration.

At the same time, other authors [2-7] believe that physical adsorption is a promising means of hydrogen storage. In particular, fullerenes, single-walled carbon nanotubes (SWNTs), carbon nanofibers and metal-organic frameworks [8] have been treated as promising adsorbents $[4,7]$. Monte Carlo simulations of hydrogen adsorption on SWNTs bunches at 77 and $293 \mathrm{~K}$, and pressures up to $20 \mathrm{MPa}[5,6]$ revealed a high enough adsorption capacity of these structures. However, until recently, the results of adsorption simulation in microporous carbon adsorbents on, SWNTs and nanofibers has not allowed one to draw a final conclusion regarding the potential use of carbon adsorbents for practical hydrogen storage. 
At the same time, there are experimental data for hydrogen adsorption in carbon materials at different temperatures and pressures [7]. As a rule, in theoretical estimations and computer simulations, the porous adsorbents under investigation are represented as a single slit-like pore of the width $H$ corresponding to an average adequate value of the pore size in real carbon adsorbents.

Basic theoretical approaches to adsorption in porous materials are based on semiempirical state equations of adsorption layers, in particular on the Langmuir isotherm, the Dubinin-Radushkevich and Dubinin-Astakhov equations [9, 10]. Among more modern approaches, the vacancy solution theory [11] and the classical density functional theory (DFT) [12-14] seem to be of special interest. Monte-Carlo simulations [15,16] should also be also mentioned as one of the available approaches for investigating the adsorption of hydrogen in different types of carbon adsorbents. However, Monte Carlo results for slit-like micropores formed by graphene hexagonal planes do not give a clear answer on the potential of microporous carbon adsorbents for the hydrogen storage [16].

In our previous papers [17-20], DFT was used to calculate the adsorption of hydrogen on fullerene molecules and in the slit-like pore formed by graphene walls. The slit-like pore reproduces, to a greater or lesser extent, conventional industrial carbon adsorbents. In [20] two important conclusions were made:

(i) at high temperatures $(T>200 \mathrm{~K})$ the adsorption $a$ per $1 \mathrm{~g}$ of adsorbent and the gravimetric density $w t$ for the cases of the available industrial carbon adsorbents and fullerene molecules are not sufficient for the practical design of the adsorption hydrogen accumulator (according our estimations, $a \approx 20 \mathrm{mmole} / \mathrm{g}$ and $w t \approx 5 \%$ );

(ii) fullerenes and fullerene materials hardly have any advantages in comparison with conventional carbon adsorbents.

In spite of many opinions that an appropriate hydrogen accumulator, including adsorption one, cannot be designed, publications on this topic have not ceased. For instance, in [21] some electrospun composite nanofibers containing carbon nanotubes were fabricated and investigated. However, at high temperatures, the authors of [21] experimentally obtained approximately the same adsorption characteristics as our DFT calculation results for carbon modelling adsorbents and fullerene molecules [20]. So, additional more accurate experimental and theoretical investigations are needed to solve the problem or to show that it principally cannot be solved. In particular, we do not believe that the adsorption and gravimetric density at $T=77 \mathrm{~K}$ and at $T=200 \mathrm{~K}$ can be approximately equal as it is stated in $[22]$.

\section{Method of calculations}

DFT theory may be regarded as a powerful modern method for the investigation of vapor-liquid interfaces, adsorption in pores, and calculations of pore size distributions from experimental isotherms. As the hydrogen molecule is nonpolar and has very low degree of asphericity, molecular hydrogen belongs, in a reasonable approximation, to the class of simple fluids. Respectively, theoretical results obtained in the framework of the simple fluid model can be correctly compared with corresponding experimental data. However, a detailed comparison of DFT results with experimental data is not straightforward. Structural and energetic heterogeneity of pores strongly influences on the adsorption in real materials [23,24]. The ways of measurements are different and, therefore, some experimental results contradict to each other [25]. Hence, there are reasons to calculate average values of the adsorption for adequate modelling systems and to compare them with the available experimental data. 
TABLE 1. Molecular parameters for $\mathrm{H}_{2}$ and graphite.

\begin{tabular}{lcc}
\hline & Linear parameter $\sigma, \mathrm{nm}$ & Energy parameter $\varepsilon / k, \mathrm{~K}$ \\
\hline \hline Graphite & $0.34[27]$ & $28[27]$ \\
Hydrogen & $0.296[29]$ & $36.7[29]$ \\
\hline
\end{tabular}

The slit-like pore model seems to be quite adequate, as the formation of micropores in carbon adsorbents can be considered as a process of thermochemical elimination of atoms from hexagonal planes in carbon crystallites being activated by water vapor [22]. In our calculations, we used the model of an ideal pore, for which the distance between the surfaces presented by two graphene layers corresponds the 1-5 deleted (burnt out) layers of graphene. So, the neighboring micropores will be separated by single carbon grapheme monolayer. We assume that the distance $H$ between hexagonal layers in graphite is equal to $0.335 \mathrm{~nm}$ and the side length $b$ of hexagons, forming layers, is $0.142 \mathrm{~nm}[27]$.

An adsorbate molecule located in the pore undergoes adsorptive forces of two planes (below referred to as upper and lower). The effect of the finite wall length is small enough and, respectively, may be neglected [26]. The interaction potential between a fluid molecule and one graphene wall was described by the next potential [28]:

$$
U_{s f}(z)=2 \pi \rho_{s} \varepsilon_{s f} \sigma_{s f}^{2}\left(\frac{2}{5} \frac{\sigma_{s f}^{10}}{z^{10}}-\frac{\sigma_{s f}^{4}}{z^{4}}\right),
$$

where $\rho_{s}$ is the wall surface density equal to the number of atoms per unit area, $\sigma_{s f}$ and $\varepsilon_{s f}$ are the parameters of the wall-fluid potential. The adsorption (single particle) potential (1) was obtained integrating the Lennard-Jones (LJ) pair potential over the wall area. For this reason, it will be referred to as the integrated LJ-potential (ILJ). The wall-adsorbate parameters $\sigma_{s f}$ and $\varepsilon_{s f}$ were found using the Lorentz-Berthelot mixing rules, via the values of $\sigma_{s}, \varepsilon_{s}$ for the solid walls and $\sigma_{f}, \varepsilon_{f}$ for the fluid, respectively, i.e. $\sigma_{s f}=\left(\sigma_{s}+\sigma_{f}\right) / 2$ and $\varepsilon_{s f}=\sqrt{\varepsilon_{s} \varepsilon_{f}}$. The reduced surface density of atoms in the walls may be evaluated as follows:

$$
n_{s}=\rho_{s} \sigma_{s}^{2}=\frac{N_{s}}{S} \sigma_{s}^{2}=\frac{4 \sigma_{s}^{2}}{3 \sqrt{3} b^{2}}=\frac{4 \sqrt{3}}{9}\left(\frac{\sigma_{s}}{b}\right)^{2}=4.413
$$

So, the total potential of the pore walls

$$
U(z)=U_{s f}(z)+U_{s f}(H-z)
$$

is equal to the sum of two terms corresponding to two walls located at $z=0$ and $z=H$, respectively, where $z$ is the distance between the fluid particle and the first wall. All the used parameters of the potential (1) are presented in Table 1 ( $k$ is the Boltzmann constant).

The main problem of DFT is the design of thermodynamic characteristic functions as some density functionals. For this purpose, expressions for the Helmholtz energy $F[\rho(\mathbf{r})]$ and grand potential $\Omega[\rho(\mathbf{r})]$ of the system under consideration are used as the density functionals. The expression for $\Omega[\rho(\mathbf{r})]$ can be written as [14]

$$
\Omega[\rho(\mathbf{r})]=F[\rho(\mathbf{r})]+\int[U(\mathbf{r})-\mu] \rho(\mathbf{r}) d^{3} \mathbf{r},
$$

where $\mu$ is the chemical potential. The Helmholtz energy functional can be formally expressed as the ideal-gas term

$$
F_{i d}[\rho(\mathbf{r})]=k T \int[\ln \rho(\mathbf{r})-1] \rho(\mathbf{r}) d^{3} \mathbf{r}
$$


and an excess contribution which, in turn, can be decomposed into the hard sphere (subscript $h s$ ) and attractive (subscript att) terms:

$$
F_{e x}[\rho(\mathbf{r})]=F_{h s}[\rho(\mathbf{r})]+F_{a t t r}[\rho(\mathbf{r})] .
$$

In Eq. (5), the cube of the thermal wavelength $\Lambda$ is omitted because it gives no direct contribution to the density profiles or thermodynamic properties. The excess Helmholtz energy term, due to hard sphere repulsion, will be presented in accordance with the modified fundamental measure theory [30]:

$$
F_{h s}[\rho(\mathbf{r})]=k T \int \Phi^{h s}\left[n_{\alpha}(\rho(\mathbf{r}))\right] d^{3} \mathbf{r} .
$$

In Eq. (7), the reduced excess energy density $\Phi^{h s}$ is interpreted as a function of six weighted densities $n_{\alpha}(\mathbf{r})=\int \omega^{\alpha}\left(\left|\mathbf{r}^{\prime}-\mathbf{r}\right|\right) \rho\left(\mathbf{r}^{\prime}\right) d^{3} \mathbf{r}^{\prime}$. The expressions for $n_{\alpha}$ and $\omega^{\alpha}$ are presented in [30]. The attractive part of the excess Helmholtz energy can be written in terms of the mean-field approximation:

$$
F_{\text {attr }}=\frac{1}{2} \iint u^{a t t r}\left(\left|\mathbf{r}^{\prime}-\mathbf{r}\right|\right) \rho\left(\mathbf{r}^{\prime}\right) \rho(\mathbf{r}) d^{3} \mathbf{r}^{\prime} d^{3} \mathbf{r}
$$

In Eq. (8) $u^{\text {attr }}$ is the long-range attraction part of LJ potential represented according to the WCA potential model [31]. The minimization of the grand potential with respect to the density profiles yields the following Euler-Lagrange equation

$$
\rho(\mathbf{r})=\exp \left[\frac{1}{k T}\left(\mu-U(\mathbf{r})-\frac{\delta F[\rho(\mathbf{r})]}{\delta \rho(\mathbf{r})}\right)\right]
$$

satisfied by the equilibrium density profile. Below we also use the reduced value of the local density $\eta=\pi \rho d_{f}^{3} / 6$. Here, $d_{f}$ is the hard sphere diameter of the hydrogen molecular which can be expressed though the $\sigma_{f}$ parameter by the next expression [32]

$$
d_{f}=\frac{1+0.2977 k T / \varepsilon_{f}}{1+0.33163 k T / \varepsilon_{f}+1.0477 \times 10^{-3}\left(k T / \varepsilon_{f}\right)^{2}} \sigma_{f}
$$

Knowing the density profile $\eta(z)$, one can find the reduced absolute adsorption

$$
\Gamma^{*}=\int_{0}^{H} \eta(z) d z
$$

in the pore under consideration. Below, the next commonly used adsorption parameters will be calculated:

(i) the adsorption of hydrogen (volume density)

$$
a=\frac{6}{\pi}\left(\frac{\sigma_{s}}{d}\right)^{2} \frac{\Gamma^{*}}{M_{s} \rho_{s} \sigma_{s}^{2}}
$$

in $\mathrm{kg} / \mathrm{m}^{3}$;

(ii) the gravimetric density (mass ratio)

$$
w t=\frac{m_{s}}{m_{s}+m_{f}} 100 \%=\frac{1}{1+1 /\left(a M_{f}\right)} 100 \%
$$

of $\mathrm{H}_{2}(\%)$. Here $M_{s}$ and $M_{f}$ are the molar masses of graphite and hydrogen respectively, $m_{f}$ is the total mass of hydrogen in the pore and $m_{s}$ is the mass of the adsorbent. 


\section{Calculation results}

Previously, [17-20] we have calculated the density distributions in different modeling fluid layers in the slit-like pore, for different wall parameters, external pressures and temperatures $(20.35,77$ and $200 \mathrm{~K})$. In this work, our DFT results for hydrogen adsorption in porous carbon at 20.35, 77, 200 and $300 \mathrm{~K}$, and various pressures are presented. So, all the former data have been recalculated for wider temperature and pressure ranges. Previously, we calculated the density distribution profiles corresponding to different wall parameter values, in particular for a wide range of the wall surface densities $n_{s}$ (from 2.0 to 6.2). It is also worth mentioning that an intermediate value $n_{s}=4.4$ corresponds to real porous graphite adsorbents [22].

In [22], the adsorption of hydrogen was calculated using a semi-empirical approach based on the Dubinin-Radushkevich equation. Namely, standard data for the adsorption of benzene were recalculated for the hydrogen-AC35 system. The abbreviation AC35 taken from [22] corresponds to a model porous carbon adsorbent with 3 of 5 graphene layers deleted. In the present paper, the above-mentioned adsorption characteristics were calculated for different $N$ values for deleted graphene monolayers and, in particular, for $N=3$ as the slit-like pore, corresponding to $N=3$ and $n_{s}=4.4$, which reproduces the model adsorbent AC35 most adequately.

In figure 1(a) the density profile is presented for the hydrogen layer when $N=3$, $n_{s}=4.4$ and $T=20.35 \mathrm{~K}$, corresponding to the boiling point of hydrogen. The figure demonstrates two reasonably high maxima at the pore walls, symmetrical relative to the pore center and two additional lower but noticeable maxima in the central part of the pore.

In figures 1(b) and 1(c), density profiles are shown for higher (supercritical) temperatures. Figure 1(b) corresponds to a supercritical but low enough temperature $T=77$ K, i.e. to the nitrogen boiling temperature, and a wide range of the pressures: from 0.05 to $20 \mathrm{MPa}$. One can see that at the lowest pressure $0.05 \mathrm{MPa}$ only two weak maxima at the pore walls are seen, i.e. the density profile corresponds to two density profiles at two separate graphene layers. At $p=0.625 \mathrm{MPa}$, a slope central maximum is formed with a plateau, and only at high pressures of about $6 \mathrm{MPa}$ are two pronounced central maxima formed. In figure 1(c) three density profiles are compared, corresponding to three different temperatures: 77, 200 and $300 \mathrm{~K}$ and a very high external pressure $20 \mathrm{MPa}$. One can see that, in spite of the high pressure, at temperatures 200 and $300 \mathrm{~K}$, central maxima are not observed and two maxima at the pore walls are 5-10 times smaller than at $T=77 \mathrm{~K}$. The known density profiles make it readily possible to find the adsorption $a$ and the gravimetric density $w t$. The dependences of the $a$ and $w t$ parameters upon the external pressure $p$ are shown in figures 2 and 3 for a low $(77 \mathrm{~K})$ and high $(200 \mathrm{~K})$ temperatures, respectively.

Figures 2 and 3 demonstrate the dependence of $a$ and $w t$ on the pore width $H$ or the corresponding number $N$ of the deleted graphene layers. As one could expect, increasing the pressure $p$ results in an increase of both $a$ and $w t$ parameters. At the same time, the variation of $N$ affects $a$ and $w t$ parameters in opposite directions: increasing $N$ results in a decreasing of the adsorption $a$ and an increase in the gravimetric density $w t$.

\section{Analysis of the results and discussion}

According to [33], for 2010 U.S. Department of Energy (DOE) proclaimed goals are as follows: $w t=6.5 \%$ mass ratio and $a=62 \mathrm{~kg} / \mathrm{m}^{3}$ volume density. Obviously, these goals relate to the problem of the hydrogen accumulator design. Later, the above goals were deemed to be unrealistic and, therefore, to 2015 DOE established new goals as $w t=5.5 \%$ and 


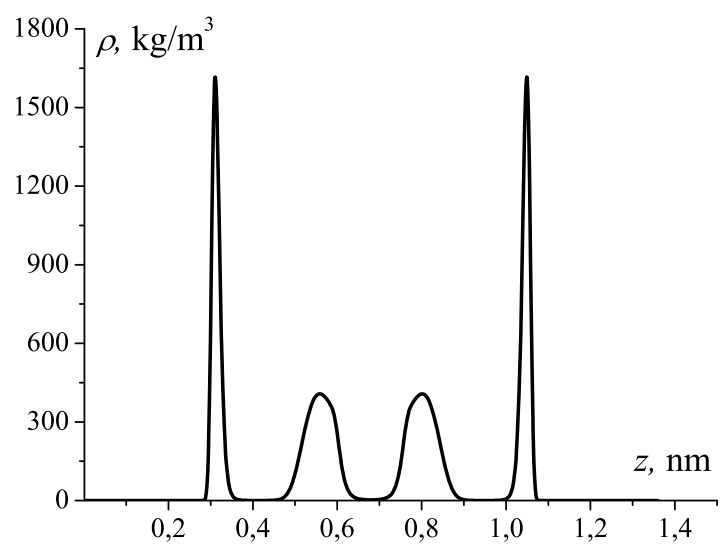

(a)

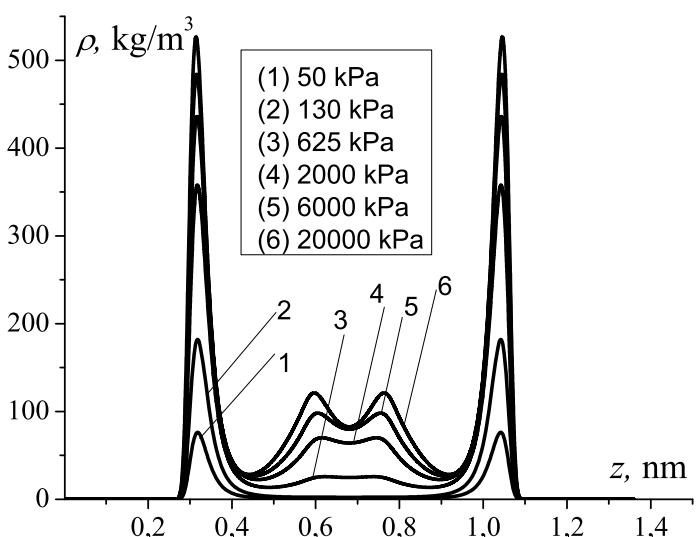

(b)

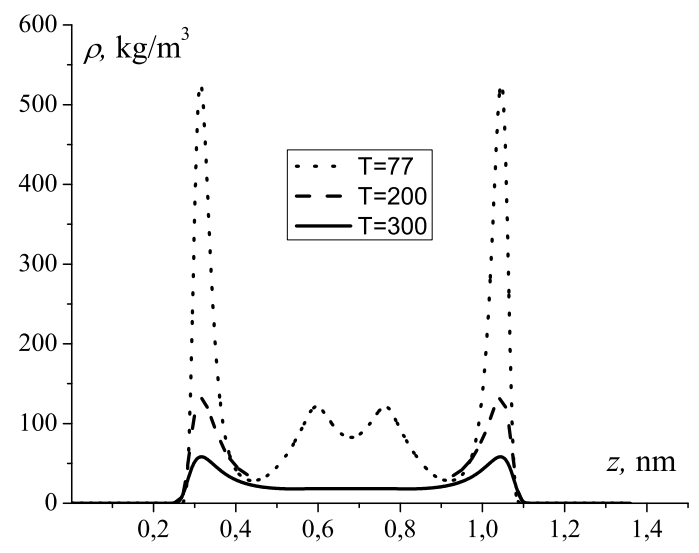

(c)

FIG. 1. Density profiles for the hydrogen layers in modeling slit-like pores representing the carbon adsorbent AC35 at different temperatures and pressures: (a) $T=20.35 \mathrm{~K}, p=85.5 \mathrm{kPa}$; (b) $T=77 \mathrm{~K}$, values of the pressure are shown in the figure; (c) comparison of the density profiles at $T=77 \mathrm{~K}, 200 \mathrm{~K}, 300 \mathrm{~K}$ and $p=20 \mathrm{MPa}$

$a=45 \mathrm{~kg} / \mathrm{m}^{3}$ at ambient temperatures and practical pressures (usually less that $10 \mathrm{bar}=1$ $\mathrm{MPa}$ ). However, according to our calculation results presented in figure 3 , at $T=200 \mathrm{~K}$ and even enormous pressures up to $25 \mathrm{MPa}$ these values of both $w t$ and $a$ parameters cannot be reached.

To confirm the reliability of our results, we compared them with the available experimental and theoretical results of other authors. In figure 4, our results for $w t$ at $T=77$ $\mathrm{K}$ are compared to experimental results [34] where a redefined pore thickness $\tilde{H}=H-\sigma_{s}$ was used as a pore parameter. The value $\tilde{H}=0.5 \mathrm{~nm}$ corresponds to $H=0.84 \mathrm{~nm}$, i.e. to $N=1.235$. Obviously, in [34] an average (effective) value of $\tilde{H}$ was used corresponding to a real carbon adsorbent. As one can see, our calculation results satisfactorily agree with the experimental results [34]. For $p=0.02 \mathrm{MPa}$ our results and results of measurements [34] coincide almost identically.

In figure 5 our results for the adsorption $a$ are compared to Monte-Carlo results [35]. As in [35], the value $\varepsilon_{f} / k=34.2 \mathrm{~K}$ was used which differs a bit from the value presented in Table 1, we also used (in this case) the same value of the $\varepsilon_{f} / k$ parameter. One can see that figure 5 demonstrates a good agreement between our calculation results and the results [35]. 


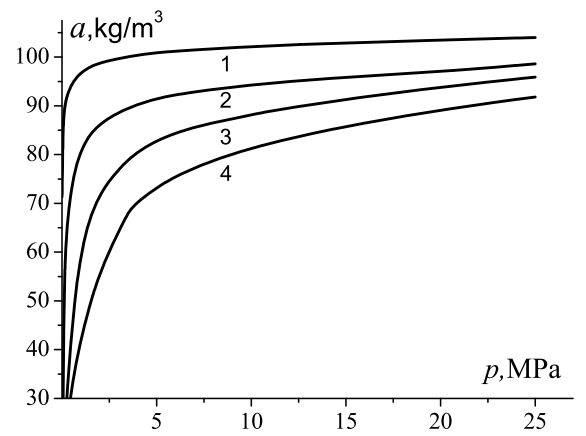

(a)

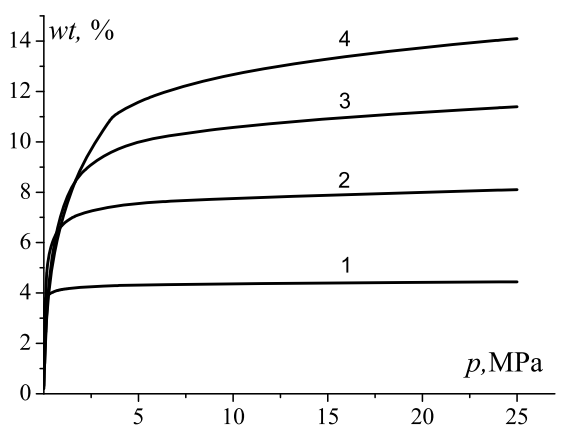

(b)

FIG. 2. The pressure dependences of the adsorption parameters $a$ (a) and $w t$ (b) at different values of $N(T=77 \mathrm{~K})$. Curves $1,2,3$ and 4 correspond to $N=1(H=0.68 \mathrm{~nm}), N=2(H=1.02 \mathrm{~nm}), N=3(H=1.36 \mathrm{~nm})$ and $N=4(H=1.7 \mathrm{~nm})$, respectively

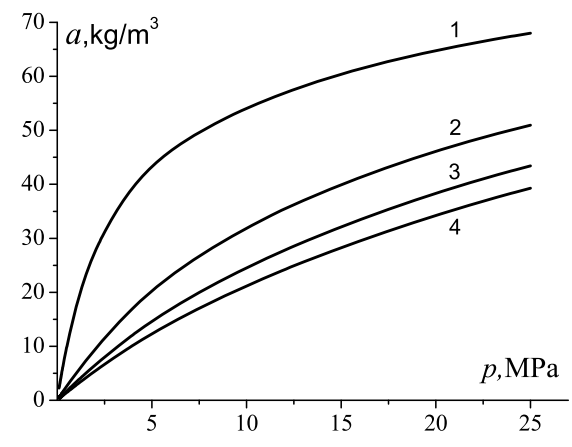

(a)

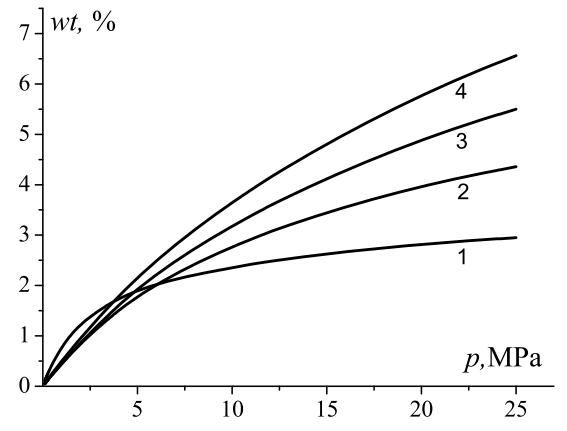

(b)

Fig. 3. The pressure dependences of the adsorption parameters $a$ (a) and $w t$ (b) at different values of $N(T=200 \mathrm{~K})$. The numeration of curves is the same as in figure 2

As was mentioned above, in [22], standard data for the adsorption of benzene in AC35 were recalculated to the adsorption of hydrogen using the Dubinin-Radushkevich equation [9]. In figure 6, our calculation results for the $a$ parameter are compared to the results of [22]. One can see that at $T=77 \mathrm{~K}$, our results satisfactorily agree with the results of [22]. However, at higher temperatures (200 and $300 \mathrm{~K}$ ), the divergence between our results and [22] becomes noticeable enough. Obviously, at 200 and $300 \mathrm{~K}$, the results for [22] are overestimated. In particular, at high pressures (on the order of $25 \mathrm{MPa}$ ) the results [22] correspond very closely to seemingly improbable $a$ values.

Another important problem which is worth discussing here is the choice of the single particle potential and its influence on the DFT calculation results. In the previous section, we used the integral form (1) of the LJ-pair potential with reliable parameters presented in Table 1. We also tried to use some ab initio data for the $\mathrm{H}_{2}$ - graphene interaction to obtain corresponding adsorption potential. However, as one can see in figure 7, different 


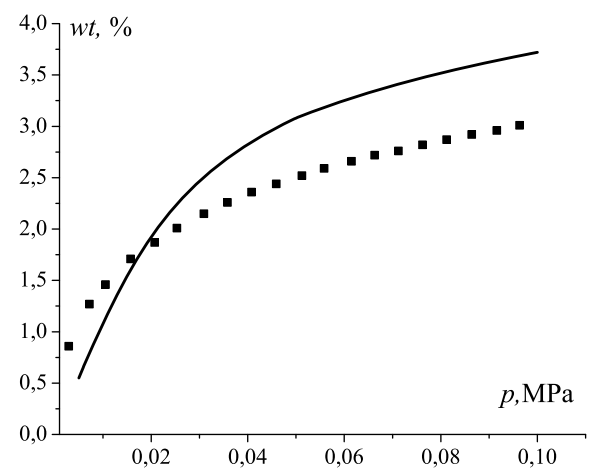

FIG. 4. Comparison of our results for the wt dependence with experimental results [34] presented by dots $\mathbf{\square}(T=77 \mathrm{~K}$, $H=0.84 \mathrm{~nm})$.

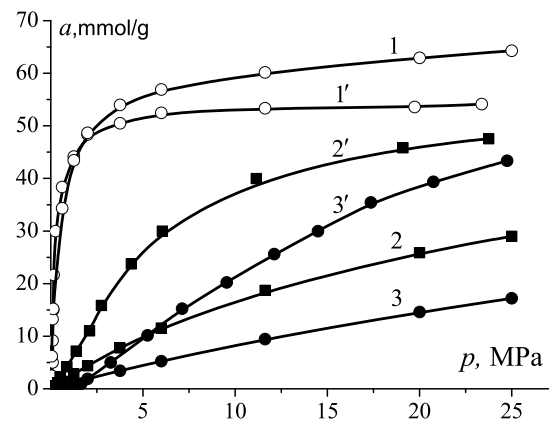

FIG. 6. Comparison of our DFT results (curves 1, 2 and 3) to the results [22] (curves 1', 2' and 3') for temperatures $77 \mathrm{~K}$ (white circles o), $200 \mathrm{~K}$ (black squares $\square$ ) and $300 \mathrm{~K}$ (black circles $\bullet$ ).

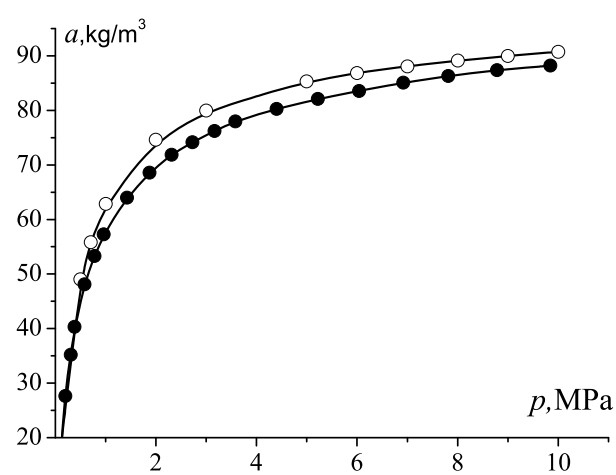

FIG. 5. Comparison of our calculation results (white circles o) to the Monte-Carlo results [35] presented by black circles • $(T=77 \mathrm{~K}, H=1.26 \mathrm{~nm}$, i.e. $N=2.7)$.

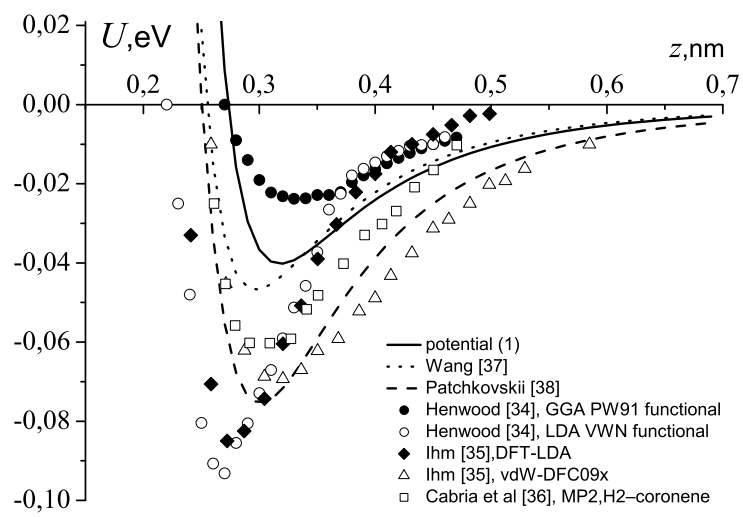

FIG. 7. Comparison of our adsorption potential (1), i.e. ILJ with ab initio data [36-38]. and semi-empirical potentials obtained via integration of $\mathrm{H}_{2}-\mathrm{C}$ pair potentials [39, 40].

approximations of the same $a b$ initio method can give noticeably different results. Also, in the available papers on the $\mathrm{H}_{2}$ - graphene interaction, only separate values of the attractive part of $U_{s f}(z)$ are presented, which makes it difficult to obtain an analytical form of $U_{s f}(z)$ suitable for further classical DFT-calculations.

It is also noteworthy that ILJ potential (1) satisfactorily agrees with an integral form of the pair potential [39]. Among the available and formally approved, to a greater or lesser extent, semi-empirical pair potentials, proposed to describe the $\mathrm{H}_{2}-\mathrm{C}$ interactions, the pair 
TABLE 2. Comparison of $a$ and $w t$ parameters calculated using ILJ and IP single particle potentials.

\begin{tabular}{|c|c|c|c|c||c|c|c|c|}
\hline \multirow{3}{*}{$N$} & \multicolumn{3}{|c||}{$T=150 \mathrm{~K}, P=20$ bar } & \multicolumn{3}{c|}{$T=298 \mathrm{~K}, P=450 \mathrm{bar}$} \\
\cline { 2 - 9 } & \multicolumn{2}{|c|}{$a$} & \multicolumn{2}{|c||}{$w t$} & \multicolumn{2}{|c|}{$a$} & \multicolumn{2}{|c|}{$w t$} \\
\cline { 2 - 9 } & ILJ & IP & ILJ & IP & ILJ & IP & ILJ & IP \\
\hline \hline 1 & 57.1 & 93.9 & 2.5 & 4.0 & 53.8 & 81.4 & 2.4 & 3.5 \\
2 & 22.7 & 66.1 & 2.0 & 5.6 & 41.9 & 62.9 & 3.6 & 5.3 \\
3 & 14.1 & 42.8 & 1.9 & 5.4 & 37.9 & 52.7 & 4.8 & 6.6 \\
4 & 11.0 & 32.1 & 1.9 & 5.4 & 35.8 & 47.1 & 6.0 & 7.8 \\
5 & 9.4 & 26.2 & 2.1 & 5.5 & 34.6 & 43.6 & 7.2 & 8.9 \\
\hline
\end{tabular}

potential [40] seems to have the deepest potential well and, therefore, may be expected to give the highest adsorption characteristics. As one could expect, the potential [40] gives also the deepest single particle potential $U_{s f}(z)$ (see figure 7).

So, we calculated the $a$ and $w t$ parameters for both ILJ and the integral form of Patchkovskii's potential (IP). The results of these calculations are presented in Table 2. As one would expect, IP really gives noticeably higher values for $a$ and $w t$ in comparison to ILJ. However, at high (room) temperature, $298 \mathrm{~K}$, the deepest IP only gives practically suitable $a$ and $w t$ values at very high pressures, which are unlikely to be realized in vehicles.

There are no reasons to consider IP as the most exact and reliable adsorption potential. Moreover, according to the results of comparison of our DFT calculations of $a$ and $w t$ parameters with the results of other authors, a conclusion can be made that IP seems to give overestimated results for the adsorption of hydrogen in carbon adsorbents. But even this deepest potential does not demonstrate some realistic opportunities for high-temperature hydrogen storage in currently-available carbon adsorbents.

\section{Conclusion}

The above comparison with the available experimental and theoretical results of other authors confirms the adequacy of our DFT calculations for the adsorption of hydrogen in carbon adsorbents. At the same time, our results demonstrate that at high (room) temperatures the adsorption capacity of carbon adsorbents is not sufficient for the practical design of an appropriate hydrogen accumulator which can be, in particular, used in vehicles. Then, according to our former results [18-20] and the results of other authors [21,41], neither conventional carbon adsorbents nor fullerenes and carbon nanotubes can help to solve this problem. This conclusion seems to be fulfilled not only for slit-like pores but also for pillared graphene [42]. At the same time, according to Monte-Carlo calculations presented in [42], the gravimetric density for Li-doped pillared materials can be several times higher than that of the analogous pure carbon materials. However, the very significant role of active sites in carbon adsorbents should be verified by further experimental and theoretical investigations, including classical DFT-calculations, which can be carried out, but are beyond the scope of this paper.

\section{Acknowledgements}

The work was supported by Russian Foundation for Basic Research (grant No 13-0300119) and by Ministry of Education and Science of Russian Federation, in frames of the 
State Program in sphere of scientific activity. The authors are grateful for useful discussion to Prof. E.A. Ustinov.

\section{References}

[1] Dillon A. C., Jones K. M., Bekkedahl T. A., Kiang C. H., Bethune D. S., Heben M. J. Storage of hydrogen in single walled carbon nanotubes. Nature, 1997, 386, P. 377-379. doi:10.1038/386377a0

[2] Hynek S., Fuller W., Bentl J. Hydrogen storage by carbon sorption. J. Int. J. Hydrogen Energy, 1997, 22, P. 601-610. DOI: 10.1016/S0360-3199(96)00185-1

[3] Carpetis C., Peschka W. Study on hydrogen storage by use of cryoadsorbents. Int. J. Hydrogen Energy, 1980, 5, P. 539-554. DOI: 10.1016/0360-3199(80)90061-0

[4] Chambers A., Park C., Baker R. T., Rodriguez N. M. Hydrogen Storage in Graphite Nanofibers. J. Phys. Chem. B., 1998, 102, P. 4253-4256. DOI: 10.1021/jp980114l

[5] Darkrim F., Levesque D. Monte Carlo simulations of hydrogen adsorption in single-walled carbon nanotubes. J. Chem. Phys., 1998, 109, P. 4981-4984. http://dx.doi.org/10.1063/1.477109

[6] Yin Y. F., Mays T., McEnan B. Molecular Simulations of Hydrogen Storage in Carbon Nanotube Arrays. Langmuir, 2000, 16, P. 10521-10527. DOI: 10.1021/la000900t

[7] Anson A., Jagiello J., Parra J. B., Sanjuan M. L., Benito A. M., Maser W. K., Martinez M. T. Porosity, Surface Area, Surface Energy, and Hydrogen Adsorption in Nanostructured Carbons. J. Phys. Chem. B., 2004, 108, P. 15820-15826. DOI: 10.1021/jp047253p

[8] Assfour B. Thaer assaad, adnan odeh, Hydrogen adsorption properties of metal-organic frameworks within the density-functional based tight-binding approach. Nanosystems: physics, chemistry, mathematics, 2014, 5, P. 820-828. http://nanojournal.ifmo.ru/en/wpcontent/uploads/2014/12/NPCM56P820.pdf

[9] Dubinin M. M. Physical Adsorption of Gases and Vapors in Micropores, in: D. A. Cadenhead (ed.), Progress in Surface and Membrane Science. City.:New York Acad. Press, 1975, 1-70 p.

[10] Ismadji S., Bhatia S. K. A Modified Pore-Filling Isotherm for Liquid-Phase Adsorption in Activated Carbon. Langmuir, 2001, 17, P. 1488-1498. DOI: 10.1021/la0009339

[11] Bhatia S. K., Ding L. P. Vacancy solution theory of adsorption revisited. J. AIChE, 2001, 47, P. 21362138. DOI: $10.1002 /$ aic.690470924

[12] Neimark A. V., Ravikovitch P. I. Calibration of Pore Volume in Adsorption Experiments and Theoretical Models. Langmuir, 1997, 13, P. 5148-5160. DOI: 10.1021/la970266s

[13] Ravikovitch P. I., Vishnyakov A., Russo R., Neimark A. V. Unified Approach to Pore Size Characterization of Microporous Carbonaceous Materials from $\mathrm{N}_{2}, \mathrm{Ar}$, and $\mathrm{CO}_{2}$ Adsorption Isotherms. Langmuir, 2000, 16, P. 2311-2320.

[14] Wu J. Density functional theory for chemical engineering: from capillarity to soft materials. J. AIChE, 2006, 52, P. 1169-1193. DOI: 10.1002/aic.10713

[15] Vishnyakov A., Ravikovitch P. I., Neimark A. V. Molecular Level Models for $\mathrm{CO}_{2}$ Sorption in Nanopores. Langmuir, 1999, 15, P. 8736-8742. DOI: 10.1021/la990726c

[16] Rzepka M., Lamp P., de la Casa-Lillo M. A. Physisorption of Hydrogen on Microporous Carbon and Carbon Nanotubes. J. Phys. Chem. B., 1998, 102, P. 10894-10898. DOI: 10.1021/jp9829602

[17] Samsonov V. M., Zubkov V.V., Grinev I. V. Application of density functional method to problem of creating hydrogen adsorption fuel cell. Technical Physics Letters, 2011, 37, P. 302-305. DOI: $10.1134 /$ S1063785011040134

[18] Zubkov V.V., Samsonov V.M., Grinev I. V. Study of structural and thermodynamic parameters of adsorption layers using density functional theory. Local density and adsorption isotherms on spherical surfaces. Colloid Journal, 2013, 75, P. 524-531. DOI: 10.1134/S1061933X13040194

[19] Zubkov V.V., Samsonov V.M., Grinev I. V. Hydrogen adsorption in slit-shaped pores on carbon adsorbents. Bulletin of the Russian Academy of Sciences. Physics, 2012, 76, P. 814-818. DOI: $10.3103 / \mathrm{S} 1062873812070398$

[20] Samsonov V. M., Zubkov V. V., Grinev I. V. Comparative study of hydrogen adsorption in slit like pore of carbon adsorbents and on fullerene molecules. Proceedings of the "International Conference Nanomaterials: Applications and Properties",Alushta, the Crimea (Sumy State University, Sumy, 2013), Sep. 16-21, 2013, P. 01NTF14. 
[21] Haji A., Nasouri K., Shoushtari A. M., Kaflou A. Reversible Hydrogen Storage in Electrospun Composite Nanofibers. Proceedings of the "International Conference Nanomaterials: Applications and Properties", Alushta, the Crimea (Sumy State University, Sumy, 2013), Sep. 16-21, 2013, P. 03NCNN05.

[22] Fomkin A. A., Sinitsyn V. A. Hydrogen Adsorption on Model Nanoporous Carbon Adsorbents. Protection of Metals, 2008, 44, P. 150-156. DOI:10.1134/S0033173208020070

[23] Nguyen T. X., Bhatia S. K. Probing the Pore Wall Structure of Nanoporous Carbons Using Adsorption. Langmuir, 2004, 20, P. 3532-3535. DOI: 10.1021/la036244p

[24] Bhatia S. K. Density Functional Theory Analysis of the Influence of Pore Wall Heterogeneity on Adsorption in Carbons. Langmuir, 2002, 18, P. 6845-6856. DOI: 10.1021/la0201927

[25] Fenelonov B. V., Ustinov E. A., Yakovlev V. A., Barnakov Ch. N., Melgunov M. S. Carbon Adsorbents as Candidate Hydrogen Fuel Storage Media for Vehicular Applications. Kinetics and Catalysis, 2007, 48, P. 599-602. DOI: 10.1134/S0023158407040192

[26] Vanin A. A., Piotrowskaya E. M., Brodskaya E. N. Molecular and statistical modeling of adsorption of binary mixture of Lennard-Jones fluids in graphite mesopores square section. Zhurnal fizicheskoi khimii, 2004, 78, P. 2064-2070.

[27] Do D. D. Adsorption analysis: equilibria and kinetics. City.:London Imperial College Press, 1998.

[28] Zubkov V.V., Grinev I.V., Samsonov V.M. Single-particle potentials for adsorbents with spherical and cylindrical geometry. Nanosystems: physics, chemistry, mathematics, 2013, 3, P. 52-68. http://nanojournal.ifmo.ru/en/articles-2/volume3/3-3/physics/paper04/

[29] Matyushov D. V., Schmid R. Calculation of Lennard-Jones energies of molecular fluids. J. Chem. Phys., 1996, 104, P. 8627-8638. http://dx.doi.org/10.1063/1.471551

[30] Yu Y-X., Wu J. Structures of hard-sphere fluids from a modified fundamental-measure theory. J. Chem. Phys., 2002, 117, P. 10156-10164. http://dx.doi.org/10.1063/1.1520530

[31] Weeks D. J., Chandler D., Andersen H.C. Role of Repulsive Forces in Determining the Equilibrium Structure of Simple Liquids. J. Chem. Phys., 1971, 54, P. 5237-5247. http://dx.doi.org/10.1063/1.1674820

[32] Tang Y. Role of the BarkerHenderson diameter in thermodynamics. J. Chem. Phys., 2002, 116, P. 66946700. http://dx.doi.org/10.1063/1.1461360

[33] Peng L., Morris J.R. Prediction of Hydrogen Adsorption Properties in Expanded Graphite Model and in Nanoporous Carbon. J. Phys. Chem. C., 2010, 114, P. 15522-15529. DOI: 10.1021/jp104595m

[34] Gogotsi Yu., Dash R.K., Yushin G., Yildirim T., Laudisio G., Fischer J. E. Tailoring of Nanoscale Porosity in Carbide-Derived Carbons for Hydrogen Storage. J. Am. Chem. Soc., 2005, 127, P. 16006 16007. DOI: $10.1021 /$ ja0550529

[35] Tanaka H., Noguchi D., Yuzawa A., Kodaira T., Kanoh H., Kaneko K. Quantum Effects on Hydrogen Isotopes Adsorption in Nanopores. J. Low Temp. Phys., 2009, 157, P. 352-373. DOI 10.1007/s10909009-9917-8

[36] Henwood D., Carey D. J. Ab initio investigation of molecular hydrogen physisorption on graphene and carbon nanotubes. Phys. Rev. B., 2007, 75, P. 245413-245423. DOI: 10.1103/Phys. Rev. B.75.245413

[37] Ihm Y. ,Cooper V.R., Peng L., Morris J.R. The influence of dispersion interactions on the hydrogen adsorption properties of expanded graphite. J. Phys.: Condens. Matter., 2012, 24, P. 424205-424212. DOI:10.1088/0953-8984/24/42/424205

[38] Cabria I., Lopez M. J., Alonso J. A. Hydrogen storage capacities of nanoporous carbon calculated by density functional and Moller-Plesset methods. Phys. Rev. B., 2008, 78, P. 075415-075424. DOI: 10.1103/PhysRevB.78.075415

[39] Wang S. C., Senbetu L., Woo C. W. Superlattice of parahydrogen physisorbed on graphite surface. J. Low Temp. Phys., 1980, 41, P. 611-628. http://link.springer.com/article/10.1007/BF00114368

[40] Patchkovskii S., Tse J.S., Yurchenko S. N., Zhechkov L., Heine T., Seifert G. Graphene nanostructures as tunable storage media for molecular hydrogen. PNAS, 2005, 102, P. 10439-10444. www.pnas.org/cgi/doi/10.1073/pnas.0501030102

[41] Spyrou K., Gournis D., Rudolf P. Hydrogen Storage in Graphene-Based Materials: Efforts Towards Enhanced Hydrogen Adsorption. ECS Journal of Solid State Science and Technology, 2013, 2, P. 31603169. DOI: $10.1149 / 2.018310$ jss

[42] Dimitrakis G. K., Tylianakis E., Froedakis G. E. Pillared Graphene: A New 3-D Network Nanostructure for Enhanced Hydrogen Storage. Nano Letters, 2008, 8, P. 3166-3170. DOI: 10.1021/nl801417w 